\title{
EFECTIVIDAD DEL RODENTICIDA BIORAT EN AMBIENTES PECUARIOS Y URBANO
}

\author{
Freddie Villafaña* , Reynaldo Espino**, Grisel Montero*, Juan G. Bornote*, Manuel \\ Diaz* y Nohema Alonso*
}

\section{RESUMEN}

La actividad de roedores en las madrigueras de cuatro ambientes diferentes en Cuba fue de 80 al 98\% durante el Pre-tratamiento. El biorodenticida Biorat fue distribuido a razón de $40-50 \mathrm{~g} / \mathrm{madriguera}$. Por primera viz en nuestro país se obtuvó el promedio días/muerte de los roedores sinantrópicos en condiciones naturales variando de 16,50 a 19,06 días, diferindo significativamente entre ellos $\left(\mathrm{H}=8,6^{*}\right)$. También se comprobó que no existen diferencias significativas $(G=8,27 ; p>0,05)$ entre los porcentajes de actividad residual en las madrigueras. Los resultados obtenidos demuestran que el producto es mucho mas efectivo donde existe gran población de estos vectores.

UNITERMOS: Biorodenticida, Salmonella enterica, Control de roedores.

\section{INTRODUCCION}

El control biológico es la acción de organismos fitófagos, predadores, parásitos y patógenos en el mantenimiento de otros organismos a un nivel de densidad más bajo del logrado en su ausencia (10). El control microbiológico está basado en la patogenicidad selectiva de los microorganismos o su capacidad de provocar enfermedades que conduzcan a la destrucción del hospedero, sin generar en él procesos de repulsión y defensa y que, al mismo tiempo, sean inocuos para las personas y los animales útiles.

El control microbiológico de roedores sinantrópicos fue propuesto por primera vez a finales del s. XIX por los eminentes científicos Luis Pasteur, Mechnikov, Gamaleia, continuándolo MERZHKOVSKY, ISACHENKO, BONFOI y PRÓJOROV $(2,4)$. En la actualidad en varios paises otros investigadores han desarrolado esta método.

Existen hasta el presente algunas evaluaciones económicas para conocer las pérdidas ocasionadas por los roedores, aunque éstas son escasas nos demuestran la necesidad de estudiar esta problemática. Es por eso que la lucha contra estos

Depto. Control de Vectores, Instituto de Medicina Tropical "Pedro Kouri", La Habana, Cuba.

** Instituto de Ecología y Sistemática ACC, La Habana, Cuba.

Recebido para publicação em 16/03/95 
indeseables animales tiene un gran valor higiénico-economico y una gran significación en cuanto a la profilaxis de las enfermedades que transmiten.

En América Latina y el Caribe sus daños fluctúan entre 8 y $10 \%$ en pérdidas, con valores superiores a U $\$ 1500$ millones por año y el consecuente perjuício para la economía de la región (8).

El uso de agentes microbiológicos para el control de roedores dañinos fue estudiado en Cuba durante el período de 1967-1970 por ARZUMANIAN et al. (1). Posteriormente ESPINO y colaboradores desarrollaran la tecnología para la producción, por primera vez en condiciones tropicales, del biorodenticida Biorat elaborado a partir de Salmonella enterica sub especie enterica, serotipo enteritides I-7 F-4, monopatógena específica de roedores (3).

En la actualidad el biopreparado Biorat ha sido aplicado en diversas areas infestadas con resultados satisfactorios, siendo considerado como un método más en la lucha integral contra las especies de roedores de importancia médico-sanitaria y económica.

En el presente trabajo nos propusimos valorar la eficacia del Biorat (Salmocumarin) en tres ambientes pecuarios y uno urbano.

\section{MATERIALES Y METODOS}

El trabajo se dasarrolló en cuatro ambientes: un cebadero de bovinos, dos pecuarios y uno urbano. El primero se encuentra ubicado en la provincia de Ciego de Avila, los dos pecuarios en la provincia Habana y el último en Ciudad de la Habana.

Para determinar los índices de actividad de roedores se utilizó el método indirecto que se basa en el conteo y taponamiento de las madrigueras durante el pre- y post-tratamiento (11).

En la fase de pre-tratamiento se taponearon todas las madrigueras de cada ambiente durante 5 días consecutivos. En el tratamiento seguimos el método recomendado por ICI (5) para los controles químicos, el cual fue corroborado por nosotros (13).

El rodenticida Biorat fue distribuido a razón de 40-50 g por cueva o madriguera. Además en la periferia de cada ambiente en estudio se colocaron 100 estaciones de $25 \mathrm{~g}$ del producto. Para el cálculo de la actividad residual de los roedores en la etapa de post-tratamiento utilizamos la misma metodología que en la primera fase, con la diferencia que las cuevas o madrigueras fueron tapadas a los 5, 20 y 30 días de haberse efectuado el control.
Se aplicaron, en total, $28 \mathrm{~kg}$ del biopreparado Biorat elaborado en la Empresa de Productos Veterinarios LABIOFAM en la provincia de Matanzas, con un título de 4 $\times 10^{9}$ células microbianas/g. Para la apliacación se emplearon cubos esmaltados de 10 y cucharas de $50 \mathrm{~g}$. Se distribuyó un total de 400 estaciones en los 4 ambientes. Diariamente se contaron los roedores muertos durante 30 días por zona de estudio para el calculo del promedio días/muerte, que permite estimar el tiempo medio necesario para que se produzca la muerte de los animales. Se realizó la necropsia de corazón, bazo e hígado para buscar las alteraciones anatomopatológicas causadas por la infección, tales como intestino gelatinoso, hepatomegalia y esplenomegalia. Posterivimente fueron realizados los testes microbiológicos de los órganos en al medio selectivo de Ploskiriov con el objetivo de lograr aislar Salmonella enteriditis var. Isachenko, corroborándose microscópicamente con tinción de Gram.

Cada ambiente en estudio contó con un área testigo, lo suficiente alejada y protegida de las posibles emigraciones de los roedores de la zona de trabajo, para evitar de esta forma la contaminación.

Para el análisis estadístico se utilizarón las pruebas no paramétricas Kruskal Wallis, Prueba G y el Procedimiento de Nemeyí $(p=0,05)(9)$.

\section{RESULTADOS Y DISCUSION}

La Tabla 01 muestra el porcentaje de actividad antes de efectuarse el tratamiento, la cual oscilaba entre 80 y $89 \%$, lo que indica una alta actividad de las poblaciones de roedores en las zonas de trabajo.

En la Tabla 02 se indican los promedios días/muerte calculados por nosotros en condiciones tropicales (variando de 16,50 a 19,06) los que coinciden con los obtenidos por KANDIVIN (6) y por PRÓJOROV (7) y colaboradores. El análisis de varianza no paramétrico de Kruskal-Wallis determinó que hay diferencias significativas de los promedios de días/muerte $\left(\mathrm{H}=8,60^{*}\right)$ entre los 4 ambientes. Se utilizó el procedimiento de Nemeyí con el fin de determinar entre cuales ambientes existia diferencia, encontrándose que solo difieren el solar yermo y la granja bovina Peñas Pobres. Suponemos que ésta diferencia esta basada por la estructura social que poseen los roedores, teniendo más individuos muertos en menos tiempo aquellas colonias que vivian más cerca de la fuente de alimentación. Por otra parte, no todos los miembros de una colonia o grupo consumen el rodenticida con la misma frecuencia, lo que depende de la jerarquia de cada colonia o grupo. Por lo tanto, en la média que los dominantes enfermen o mueran, el resto de la colonia tendrá más oportunidad de consumir e 
veneno, lo que pudiera también explicar la diferencia significativa entre lo solar yermo y la granja bovina.

Tabla 1- Calculo de madrigueras activas antes del tratamiento con rodenticida biológico en objetivos pecuarios y urbano.

\begin{tabular}{l|cc|c}
\hline AMBIENTE & \multicolumn{2}{|c|}{ MADRIGUERAS } & $\begin{array}{c}\% \text { DE } \\
\text { ACTIVIDAD }\end{array}$ \\
\cline { 2 - 2 } & Desactivadas & Activadas & \\
\hline $\begin{array}{l}\text { Granja Bovina\# } \beta \text { Prov.Habana } \\
\text { Granja Bovina "Peñas Pobres" }\end{array}$ & 80 & 74 & 92,5 \\
$\begin{array}{l}\text { Prov. Habana } \\
\begin{array}{l}\text { Cebadero de Bovinos "Turiguano" } \\
\text { Prov. Ciego Avila }\end{array}\end{array}$ & 112 & 110 & 80,0 \\
$\begin{array}{l}\text { Solar Yermo Ciudad de la Habana } \\
\text { TOTAL }\end{array}$ & 123 & 119 & 98,0 \\
\hline
\end{tabular}

El gráfico en la Figura 01 muestra la actividad (\%) durante las fases de pre- $y$ post-tratamientos, lo que indica que el Salmocumarin es mucho más efectivo donde hay mayor densidad de ratas y ratones. Esto ocurre por existir mayores posibilidades de contacto para producirse una epizootia, lo que coincide con los resultados de VASHKOV y colaboradores (12), que plantean que para obtener una alta efectividad con el rodenticida es primordial que exista una gran actividad de estos roedores.

VILLAFAÑA, F, ESPINO, R; MONTERO, G; BORNOTE, JG; DIAZ, M; ALONSO, N Efectividad del Rodenticida Biorat En Ambientes Pecuarios Y Urbano. Rev. Pat. Trop.24 (1 ): 11 - 19, jan/jun. 1995

Tabla 2 - Efectividad del Biorat en ambientes pecuarios y urbano.

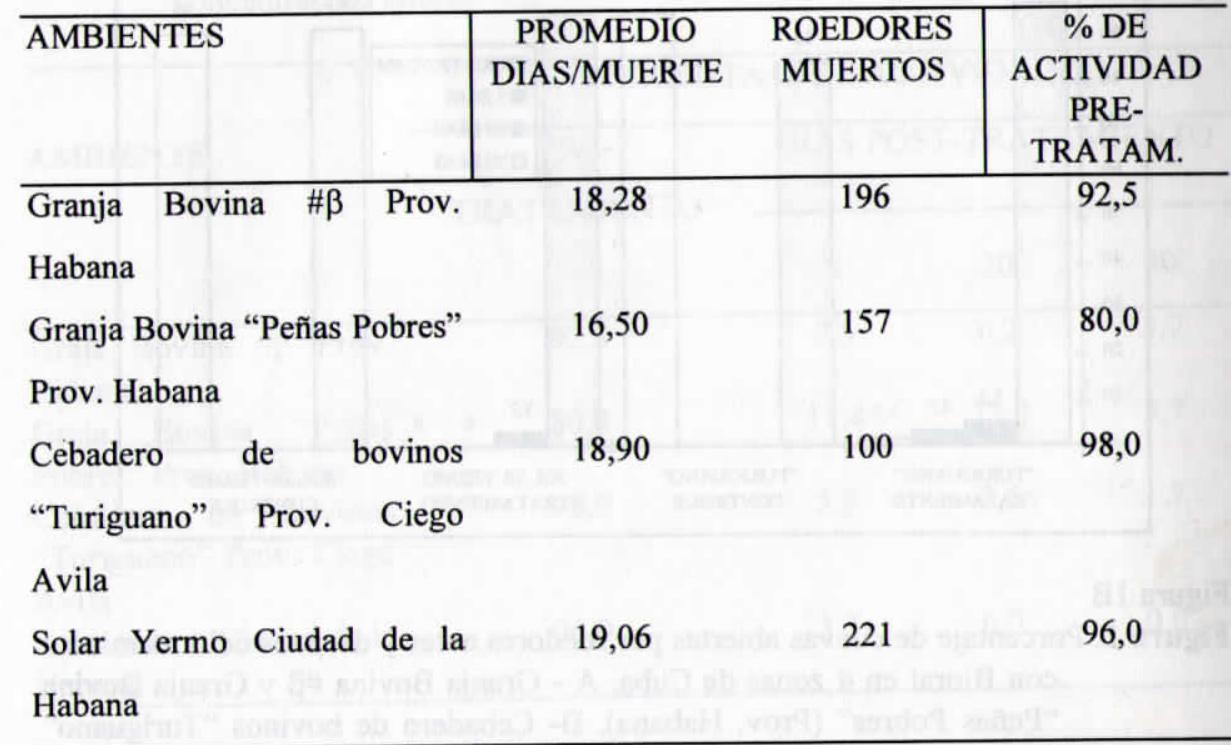

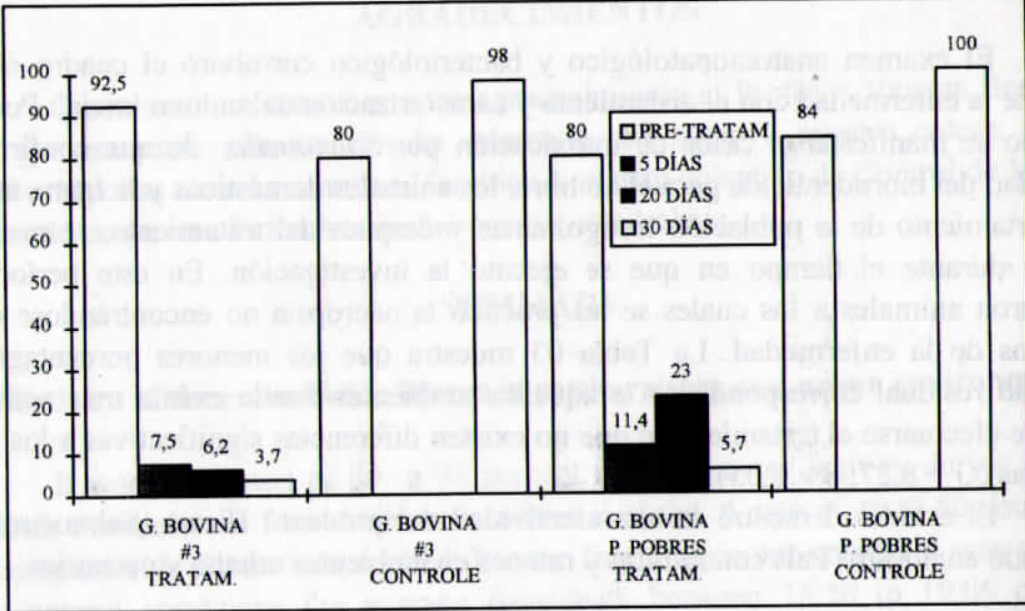

Figura $1 \mathrm{~A}$ 
VILLAFAÑA, F; ESPINO, R; MONTERO, G; BORNOTE, J.G; DIAZ, M; ALONSO, N. Efectividad del Rodenticida Biorat En Ambientes Pecuarios Y Urbano. Rev. Pat. Trop.24 (1): 11 - 19, jan/jun. 1995

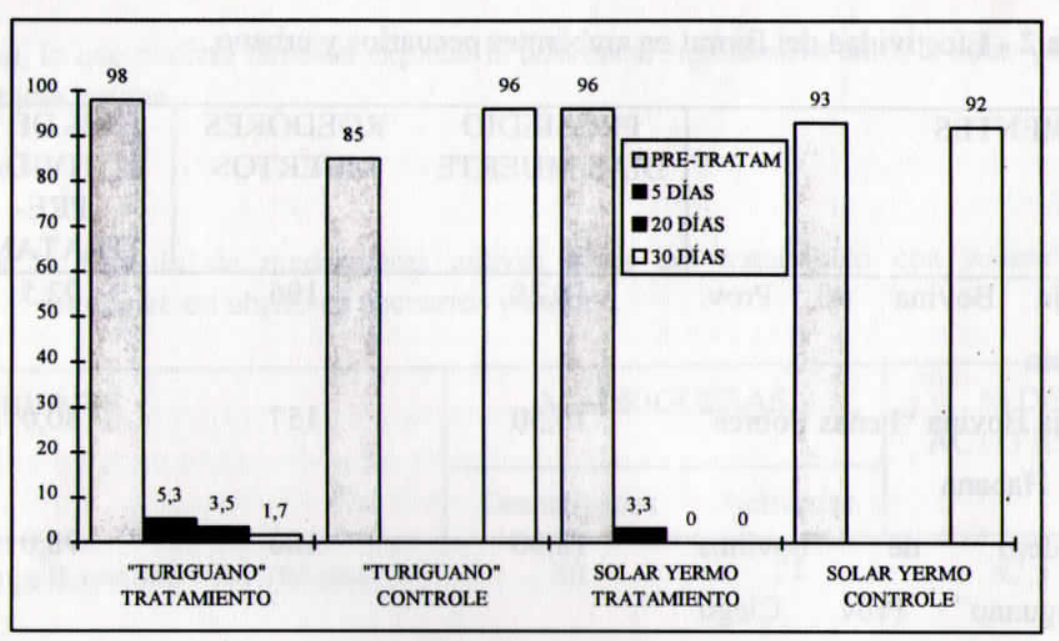

Figura 1B

Figura 1. Porcentaje de cuevas abiertas por roedores antes y despues del tratamiento con Biorat en 4 zonas de Cuba. A - Granja Bovina \# $\beta$ y Granja Bovina "Peñas Pobres" (Prov. Habana). B- Cebadero de bovinos "Turiguano" (Prov. Ciego Avila) y Solar Yermo (Ciudad de la Habana).

El examen anatomopatológico y bacteriológico corroboró el cuadro clínico típico de la enfermedad con el aislamiento y caracterización del cultivo inicial. Por otro lado, no se manifestaron casos de intoxicación por Salmonella, lo que confirma la inocuidad del biorodenticida para el hombre, los animales domésticos y la fauna útil. El comportamiento de la población testigo, antes y después del tratamiento, se mantuvo normal durante el tiempo en que se ejecutó la investigación. En este período se capturaron animales a los cuales se les practicó la necropsia no encontrándose casos positivos de la enfermedad. La Tabla 03 muestra que los menores porcentages de actividad residual correspondieron a aquellos ambientes donde existía más actividad antes de efectuarse el tratamiento e que no existen diferencias significativas a los 5, 20 y 30 días $(G=8,27 ; p>0,05)$.

El estudio demostró la alta efectividad del producto Biorat (Salmucumarin) elaborado en nuestro Pais contra ratas y ratones en ambientes urbano y pecuarios.

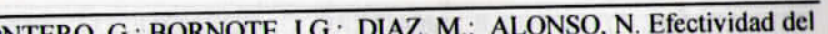
Rodenticida Biorat En Ambientes Pecuarios Y Urbano. Rev. Pat. Trop.24 (1): 11 - 19, jan/jun. 1995

Tabla 3. Porcentaje de actividad en las mdrigueras antes y despues control biologico concentrações Biorat.

\begin{tabular}{|c|c|c|c|c|}
\hline \multirow{3}{*}{ AMBIENTE } & \multicolumn{4}{|c|}{ PORCENTAJE DE ACTIVIDAD } \\
\hline & \multirow{2}{*}{$\begin{array}{c}\text { PRE- } \\
\text { TRATAMIENTO }\end{array}$} & \multicolumn{3}{|c|}{ DIAS POST-TRATAMIENTO } \\
\hline & & 5 & 20 & 30 \\
\hline $\begin{array}{l}\text { Graja Bovina \# } \beta \text { Prov. } \\
\text { Habana }\end{array}$ & 92,5 & 7,5 & 6,2 & 3,7 \\
\hline Graja Bovina "Peñas & 80,0 & 11,4 & 23,0 & 5,7 \\
\hline $\begin{array}{l}\text { Pobres" Prov. Habana } \\
\text { Cebadero de bovinos } \\
\text { "Turiguano" Prov. Ciego } \\
\text { Avila }\end{array}$ & 98,0 & 5,3 & 3,5 & 1,7 \\
\hline $\begin{array}{l}\text { Avila } \\
\text { Solar yermo Ciudad de la } \\
\text { Habana }\end{array}$ & 96,0 & 3,3 & 0,0 & 0,0 \\
\hline
\end{tabular}

\section{AGRADECIMIENTOS}

Queremos que conste nuestro agradecimiento al Profesor Vicente Berovides, quien gustosamente siempre nos ha orientado, así como a nuestro colega Agustín Tracias a ambos. También a nuestro colectivo de Control de Vectores quienes de una u otra forma han hecho posible este trabajo.

\section{SUMMARY}

\section{Effectivity of the rodenticide Biorat in cattle-raising and urban environments}

It was calculated as 80 to 98 percent the rat burrow activity during the pretreatment evaluation in four different localities in Cuba. A rate of 40 to $50 \mathrm{~g} / \mathrm{burrow}$ of treatment evaluation in four different localities in the biorodenticide Biorat was applied. For the first time in our 16,50 to 19,06 days for under natural conditions the avarage days/death between 16,50 to 19,06 days sinantropic rodents $\left(\mathrm{H}=8,6^{*}\right)$. Statistic analysis for all the four places showed indeed
no significant differences $(\mathrm{G}=8,27 ; \mathrm{p}>0,05)$ for residual burrow activity after 30 
KEYWORDS: Biorodenticide, Salmonella enterica, Rodent Control

09.SIDDNEY, S. Diseño Experimental No-Paramétrico. Ed. Rev. Instituto Cubano del Libro, La Habana, Cuba, 1970. 346 p.

10.SPRATT, D.M. The role of helminths in the biological control of mamals. Intern. Jour. Parasitol. 20(4):543-50, 1990.

\section{REFERENCIAS BIBLIOGRAFICAS}

01.ARZUMANIAN, G. \& ESPINO, R. Métodos bacteriológicos de lucha contra los roedores dañinos. ACC Instituto de Zoologia. Serie Biológica 28: 1-37, 1980.

02.BYKOVSKI, V.A. \& KANDYBIN, N.V. Biological principles, development and perspectives of the use of the bacteria and viruses. Rodent Pest Management 378-88, 1988.

03.ESPINO, L.L.R.; F. VILLAFAÑA, M.; FRAGA, J.A. \& J. BONORTE, R. Resultados de la introducción de un logro de la ciencia y la técnica y avances en la producción del bactorrodenticida Salmocumarin en la biofábrica del IMV de la provincia de matanzas durante el año 1985. I Jornada de Animales de Zoológico y Fauna Silvestre. ACC. Ciudad de La Habana, Diciembre de 1986.

04.ESPINO, L.L.R.; G. MONTERO, L.; F. VILLAFAÑA, M. \& J. BONORTE, R. Normas Técnicas para la producción de Salmocumarin en Cuba. INHEM. 1986.

05.ICI-El Rodenticida Brodifacouma. Una selección de información Publicada. ICI. Londres, Inglaterra: 1-20, 1983.

06.KANDYBIN, N.V. \& PROJOROV, M.I. Experimentos de secos de bacteria para el control de los roedores en Leningrado. Microbiologia Agrícola, 14:314-329, 1958.

07.PROJOROV, M.I. Introducción del preparado de bacterias en medios de cultivo de grano. Rev. Protec. Plantas de Plagas y Enferm., 2:64-85, 1965.

08.RODRÍGUEZ, M.J. Control de roedores en América Latina. Rev. Agricultura de las Americas. Julio/Agosto, 1992.

1.TOSCHIGUIN, Y.V. Evaluación comparativa de algunos métodos de hallazgo de ratas grises para la determinación de la efectividad de la desratización Problemas de las infecciones especialmente riesgozas. Saratov:222-4,1979.

12.VASHKOV, V.I. La lucha contra los roedores en las ciudades y centros urbanos de la localidad rural. Ed. Medicina, Moscú. 135-49, 1974.

13.VILLAFAÑA, F. Valoración biológica del rodenticida Antirat de producción nacional en poblaciones de Rattus norvegícus. Rev. Cub. Hig. y Epid. 27(4):513-19, 1989 